International Journal of Innovative Research in Science, Engineering and Technology

(An ISO 3297: 2007 Certified Organization)

Vol. 5, I ssue 8, August 2016

\title{
Design approach of Eye Tracking and Mind Operated Motorized System
}

\author{
Susmita Das ${ }^{1}$, Sayan Kumar Swar ${ }^{2}$, Shrisom Laha ${ }^{2}$, Subhankar Mahindar ${ }^{2}$, Suchetana Halder ${ }^{3}$, Koushik \\ Hati $^{2}$, Sandipan Deb ${ }^{2}$
}

Assistant Professor, Department of Electronics and Instrumentation Engineering, Narula Institute of Technology, Kolkata, West Bengal, India ${ }^{1}$

B.Tech Student, Department of Electronics and Instrumentation Engineering, Narula Institute of Technology, Kolkata, West Bengal, India ${ }^{2}$

B.Tech Student, Department of Electronics and Communication Engineering, Narula Institute of Technology, Kolkata,

West Bengal, India ${ }^{3}$

\begin{abstract}
This paper depicts the idea of design approach implementing the bio potentials generated from living body for the mobility of any motorized system needed for the physically challenged people. Here the study of eye anatomy and physiology is presented with the implementation of the bio potential generated from the body. Information about different types of bio electrodes is given and potentials generated due to eyeball movement is in the project work for the motion of the motorized system. This design approach would be useful for the disabled persons who can only move their eyeballs for the betterment of their living independently. In addition the startup of the system will be dependent upon the attention level of the disabled person thus electroencephalogram is taken under consideration. Utilizing the eye tracking method as well as electroencephalogram and reducing different sources of artifacts the generated potentials can be used to control any vehicle especially a wheelchair.
\end{abstract}

KEYWORDS: Eyeball Tracking, Corneal Retinal Potential, Electroencephalogram, Automated Motion, Bioelectrodes, Bio Feedback, LabVIEW, Neurosky, Bluetooth

\section{INTRODUCTION}

Recent technology is proceeding towards automation by using Programmable controllers and robots. In this era, an intelligent system is needed to be developed to serve the mankind. People with physical disabilities suffer from problems of mobility. New devices with sophisticated technologies help them to be comfortable. Research groups at world level have begun to give tremendous efforts to emphasize on mobility of elderly and/or disabled persons [1] for increasing their quality of life allowing them a more autonomous and independent lifestyle and greater chances of social integration. The ability to influence gaze direction of a human being can be used for movement with use of wheel chairs or motorized system. For physically challenged people with paralyzed muscles this technique will be helpful [4]. For these purposes two different types of bio potential i.e. Electro-Oculogram and Electroencephalogram is recorded. Electroencephalogram will cause the wheelchair to move forward whereas Electro-Oculogram will enable the wheelchair to change its direction accordingly. In this work LabVIEW [22] is used for the signal acquisition and processing purpose while Arduino acts as the central controller of the motorized vehicle system.

Vision controlled automation is one of the recent emerging research fields. Eye tracking is the process of tracking the movement of the eyeball to determine where the user is looking. In 1792, Wells used after images (ghost images) to describe the movement of the eyes [5]. In the 19th century, Javal (1879) and Lamare (1892) obtained audible eye 


\title{
International Journal of Innovative Research in Science, Engineering and Technology
}

\author{
(An ISO 3297: 2007 Certified Organization)
}

\section{Vol. 5, I ssue 8, August 2016}

movements using a mechanical coupling of the eyes and the ears with a rubber band. In the year 1901, Dodge and Cline made the first unobtrusive measurements of eye movements (horizontal eye movements only) using a photographic method and light reflections from the eyes [1] [5] [6].

\section{STUDY OF ELECTOOCULOGRAM}

A. Anatomy and Physiology of Eye:

The anatomy of human eye is presented in Figure 1. A hard, transparent layer, cornea forms the front of the eyeball. Surrounding this is the opaque sclera, inside of which the blood vessels form the choroid. On the front side, under the cornea, the iris extends the choroid. The iris has a round aperture in the middle called pupil. The eyeball is filled by a glasslike humor, the vitreous body. The inside of the choroid holds retina [1] [6].

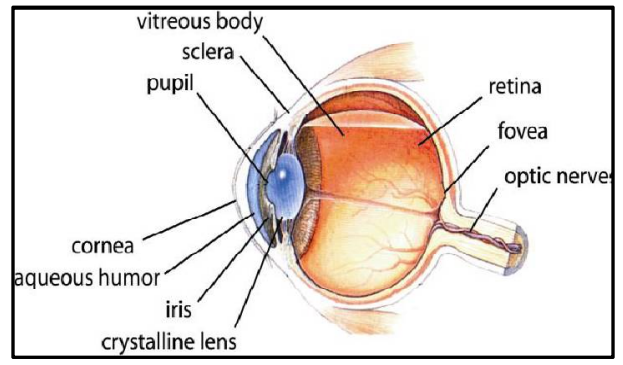

Figure 1: The structure of Human Eye [1]

The eye is rotated symmetrically by two pairs of rectus muscles and a pair of oblique muscles functioning as antagonist pairs. The lateral rectus abducts the eye toward the nose and the medial rectus abducts the eye away from the nose. These muscles move the eye in the horizontal plane. The remaining four muscles, the superior and inferior rectus (elevating and depressing the eye) and the superior and inferior oblique (controlling intorsions and extortion) control the vertical motion of the eye [2]. Thus these muscles are responsible for providing the directionality to the eyeball and allowing a total of six degrees of freedom, as shown in Figure 2, to its movement.

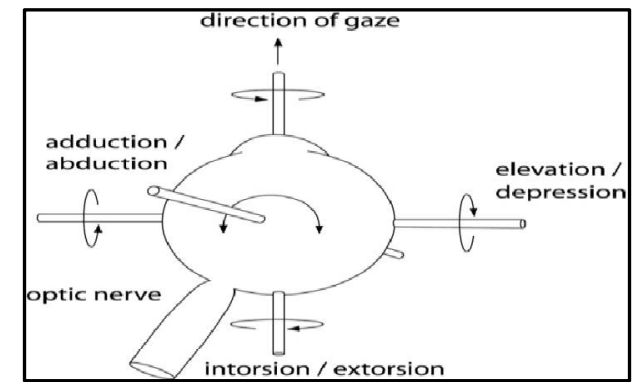

Figure 2: The Six Degrees of Freedom of The Eye [2] [6]

\section{B. Electrical Properties of the Eye:}

There is a significant difference in the metabolic rate between the corneal and retinal region. The centre of cornea is positive and the retina is negative [7]. As a result the micro-currents flow radially from the positive pole to the negative pole of the battery through the conductive tissue in the orbit. The standing potentials are generated by these currents around the eye [2]. These cornea-retinal potential can be measured and recorded using the technology known as Electro-Oculogram. 


\title{
International Journal of Innovative Research in Science, Engineering and Technology
}

\author{
(An ISO 3297: 2007 Certified Organization)
}

Vol. 5, I ssue 8, August 2016

\section{Electrooculography (EOG):}

The Electro-Oculography (EOG) method was introduced by Fenn and Hursh in 1934 [6]. It is a method for sensing eyeball movement and is based on recording the standing corneal retinal potential arising from hyperpolarization and depolarization existing between the cornea and the retina. This is commonly known as an Electro-Oculogram (EOG) [7]. The standing potentials in the eye can be measured with the voltage induced across a system of surface electrodes placed around the eyes thus making it a non-invasive process. The EOG signal is also non-repetitive.

\section{EOG Signal Acquisition and Electrode Placement}

The EOG signals are obtained by placing two electrodes to the up and down of the eyes. The EOG changes a very small microvolt for each degree of eye movement. It is necessary to eliminate the artefacts produced due to the movement of the head and the surface electrodes. To reduce the noise filtering of the signal should be processed with proper design. Simple Ag-AgCl electrodes are used [8] [10] [14]. Due to the low impedance the silicon-rubber conducting electrode is more suitable to capture the very low amplitude bio-signals as compared to other types of electrodes such as $\mathrm{Ag}-\mathrm{AgCl}$ electrodes. An electrolytic gel based on sodium chloride is applied to the skin for better conductivity as the upper layers of the skin are poor conductors of electricity.

In left gaze of the eye, the cornea approaches the electrode with a positive charge near the left eye and for right gaze, negative change in the potential difference recorded from it [2] [7]. There are three pairs of ocular muscles around the eye are superior rectus, inferior rectus, superior oblique, inferior oblique, medial rectus and lateral rectus responsible for the eye ball movement to generate electric potentials. EOG ranges from 0.05 to $3.5 \mathrm{mV}$ in humans and is linearly proportional to eye displacement and can have a frequency range of about 0.16 to $30 \mathrm{~Hz}$ [4].

\section{STUDY OF ELECTROENCEPHALOGRAM}

The recorded representation of Bio electric potentials that is generated by the neuronal activity of the brain is known as Electroencephalogram [16]. Electroencephalogram or popularly known as EEG was first used in early 20th century and since this field of biomedical instrumentation has drawn increasing attention of many researchers and scientist throughout the world. EEG is the recording of electrical brain activity through electrode sensors placed on the scalp.

\section{A. Physiology of Brain:}

The brain is a very complex architecture. Human brain mainly consists of three parts i.e. Cerebrum, Cerebellum, and Medulla Oblongata. Cerebrum is divided into different lobes i.e. Frontal, Parietal, Temporal and Occipital as can be seen in Figure 3. Cerebellum is situated to the rear under cerebrum which is also referred as small brain. Medulla Oblongata is projection of cerebellum called the brain stem and the spinal cord. Nerves carry electrical impulses along the spinal cord from the brain stem to different parts of the body and in both direction [18]. The Medulla Oblongata is generally responsible for regulating the work performed by heart and controlling blood distribution. Pons, Midbrain and Diencephalon are other essential parts of the brain. Diencephalon is then divided in two parts i.e. Thalamus which acts as a relay station for sensory pathways and Hypothalamus which acts as a centre for Temperature, Metabolism, and Fluid regulation [18].

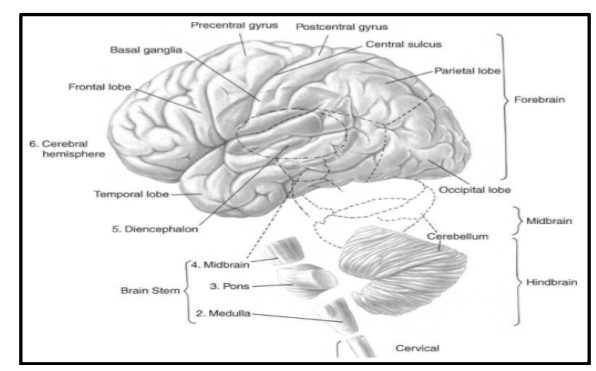

Figure 3: Brain Anatomy 


\title{
International Journal of Innovative Research in Science, Engineering and Technology
}

\author{
(An ISO 3297: 2007 Certified Organization)
}

Vol. 5, I ssue 8, August 2016

\section{B. Electrical Activity of Brain:}

The EEG potentials that is measured from the surface of the scalp is nothing but the combined effect of potentials sourced from a wide region of cerebral cortex and from various points beneath [16]. According to the modern theories electrical activity of the brain is not the summation of the action potentials of neurons in the brain rather it is the result of the graded potentials on the dendrites of the neuron in the cerebral cortex as they are influenced by other neurons that impinge on these dendrites. These graded potentials is actually a variation around the average value of the resting potentials and as these graded potentials increase above a certain threshold an action potential is developed. The amplitude of these potentials generally range between $10 \mathrm{uV}$ to $100 \mathrm{uV}$ [16] [18]. These signals can be divided into different wavebands of varying frequency in the range of 0.5 to $50 \mathrm{~Hz}$. These different wave bands are Delta $(0.5-4 \mathrm{~Hz})$ which are generated during deep sleep, Theta $(4-8 \mathrm{~Hz})$ which are generated during drowsy state, Alpha $(8-13 \mathrm{~Hz})$ which are generated during relaxation or meditative state, Beta $(13-30 \mathrm{~Hz})$ which are generated when the brain is seriously engaged in some activity and Gamma $(>30 \mathrm{~Hz})$ which is much rare and usually recorded from a child's brain activities. Due to such low amplitudes the measurement of EEG thus requires complex electronic circuitry.

\section{EEG Signal Acquisition and Electrode Placement:}

Voltage signals in range of microvolts are easily susceptible to ambient noise as well as different artefacts thus recording of such signals would require to follow proper measurement procedure. An array of surface $(\mathrm{Ag}-\mathrm{AgCl}$ electrodes) or needle electrodes is placed over the scalp following the 10-20 electrode placement system devised by International Federation of Societies for Electroencephalogram [18]. Suitable electrolyte paste is needed to be used along with the electrodes to improve coupling of these potentials. Proper amplifying and filter circuits have to be employed to amplify such low level signals and eliminate all possible noises and interferences. These can cause a lot of complexities before and during measurement as well as the entire procedure can be quite expensive. Another cost effective and efficient procedure for recording these electrical signals generating from the brain can be the use of Neurosky Mindwave headset [17]. With help of the Neurosky, by wearing a small headset one can measure the raw signals generating from his/her brains as well as the signal power spectrum. Neurosky acquires the tiny electrical voltage generated from the brain by measuring the potential difference between one's forehead (touching with sensors) and ear-lobe (clipped with ground/reference electrode). Though the limitations of recording with a single sensor with increasing chance of noise interference Neurosky can still distinguish two fairly mental states (attentive and neutral) with $85-90 \%$ accuracy [19]. This small and powerful device can thus be clubbed with applications such as health monitoring of patients in coma or mental disturbance, supporting physically disabled people and also the use of this device can be extended in the field of control instrumentation such as mind controlling of home appliances and even robots for old age robotics.

\section{METHODOLOGY OF THE PROPOSED SYSTEM}

The main objective of this project is to develop a virtual instrument that will acquire EOG as well as EEG signal and control a motorized vehicle/wheelchair wirelessly. Thus LabVIEW is used as the primary software tool to perform the acquisition, signal processing and sending the control signal to the wireless system. Atmega328 powered prototyping board Arduino is used as the central microcontroller to control the activity of the motor attached with the vehicle/wheelchair. It continuously communicates with LabVIEW through Bluetooth technology [12] [15] and acts according to the commands that it receives.

\section{A. Hardware requirement for EOG acquisition:}

The amplitude of the EOG signal is generally in few units of millivolt range [10] [18]. To make it into readable range, proper amplifying circuitry is required. AD620 IC, Instrumentation amplifier is employed as the primary amplifier due to its high gain as well as high CMRR and excellent frequency response. For proper elimination of noise a high pass filter with cut off frequency $0.16 \mathrm{~Hz}$ and a second order Low pass filter with cut off frequency $36 \mathrm{~Hz}$ is designed with help of OP07. 


\title{
International Journal of Innovative Research in Science, Engineering and Technology
}

\author{
(An ISO 3297: 2007 Certified Organization)
}

Vol. 5, I ssue 8, August 2016

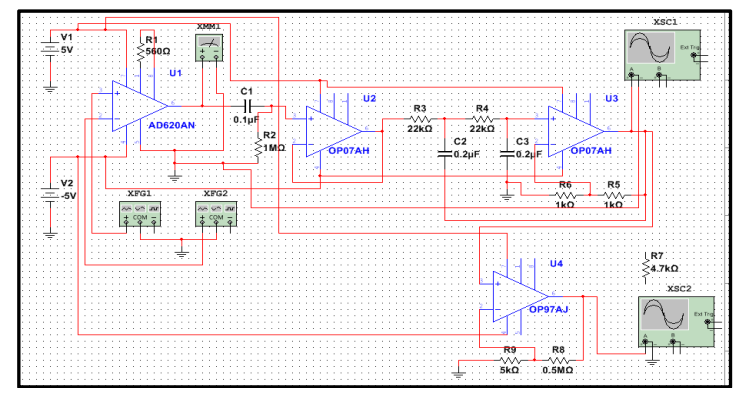

Figure 4: EOG Acquisition Circuit Diagram

For the final stage amplification OP97 op-amp is used, due to its less offset voltage as well as good amplification and decent CMRR value, with a gain value of 10 is added to bring the signal in voltage range and it is then read with the help of NIDAQ8182 and later processed in LabVIEW. The entire hardware circuitry has been designed, simulated and tested successfully in NI Multisim14, as shown in Figure 4, before implementing it in the project.

\section{B. Hardware requirement for EEG Acquisition:}

For EEG acquisition, the Neurosky Headset is an available standalone device for this complex work. Neurosky measures the signal from the forehead and wirelessly fed the data to the computer, using Bluetooth technology [20] [21], which then can be read in LabVIEW in the scale of 0-100 [19]. A proper attention level threshold can be set by the user exceeding which will further make the motorized system to move forward.

\section{Analysis in LabVIEW:}

After completion of the signal acquisition of both the types of bio signal, are analysed in LabVIEW. In this project a GUI continuously runs and communicates with the NIDAQ through USB connection and a Neurosky Mindwave Headset through Bluetooth connection. The EOG signal is read using DAQ Assistant VI with proper settings. For the filtering purpose IIR type Butterworth filter is chosen due to its linear response [9] [13]. As EOG signal may vary with person to person thus the Amplitude measurement VI is used to calculate the positive peak of the desired signal which then can be compared with a user defined threshold so that the motorized system can be calibrated to move right or left according to an individual's requirement. In parallel EEG signal from the Neurosky Mindwave is captured and analysed in LabVIEW to extract the attention level of an individual. As the attention level can vary from person to person thus a user defined threshold is allowed to set which then can be compared with the incoming signal and thus forward movement of the motorizedsystem can be controlled. The controller part of the motorizedsystem that is Arduino [23], follows the Bluetooth communication protocol to communicate with LabVIEW. It acts according to the control signal it receives thus making it a wireless and easy to implement device.

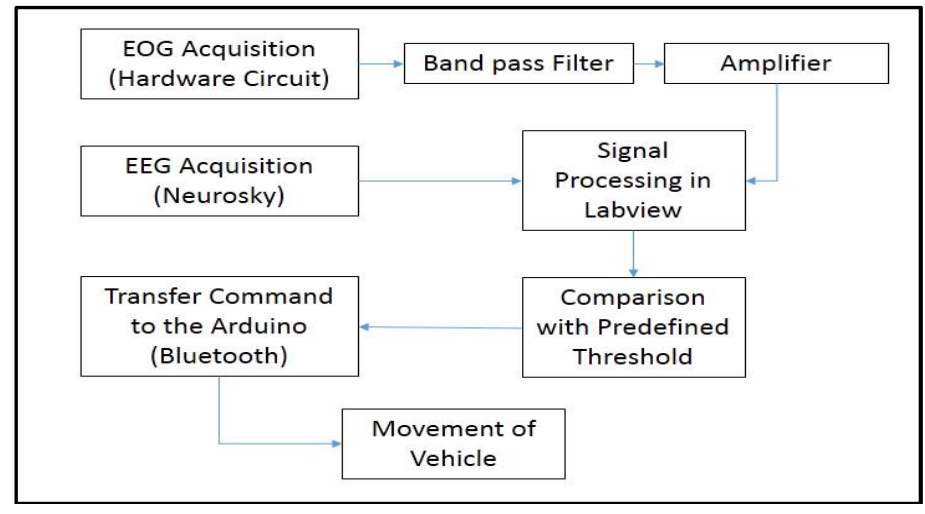

Figure 5: Block Diagram of the System 


\title{
International Journal of Innovative Research in Science, Engineering and Technology
}

\author{
(An ISO 3297: 2007 Certified Organization)
}

Vol. 5, I ssue 8, August 2016

The above Figure 5 displays the block diagram of this work. Each relation between sections like EOG and EEG acquisition, signal filtering and the control section is shown with help of the flow of control of the signal.

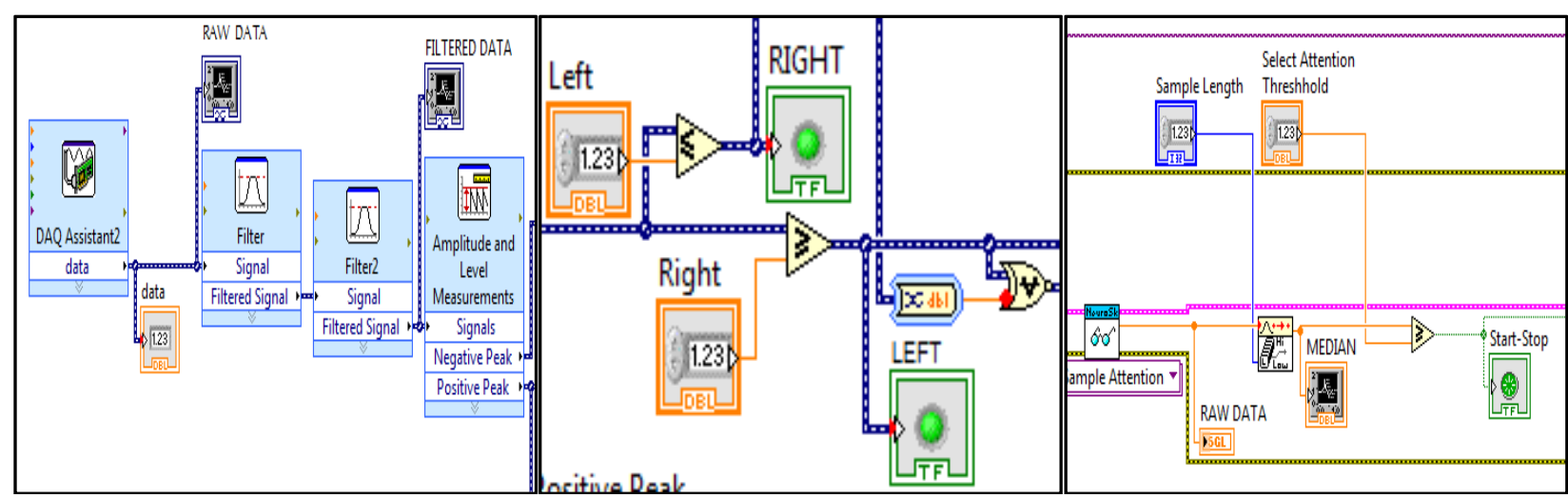

(a) (b)

(c)

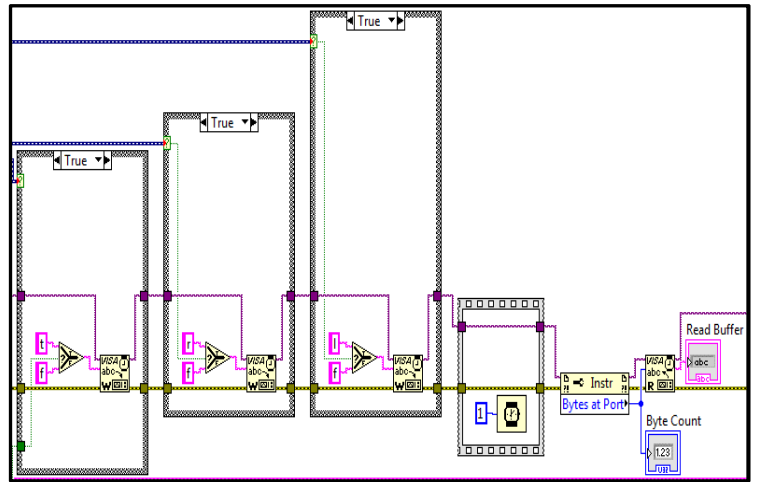

(d)

Figure 6 VI of Car Control Algorithm with Bluetooth (a)VI of EOG Acquisition and Processing (b) VI of Eyeball Tracking Algorithm (c) VI of EEG Acquisition (d)

The above figure corresponds to the LabVIEW VI sections of this work. The Figure 6 (a) indicates the raw EOG signal acquisition as well as the filtering part and extracting the positive and negative peaks of the filtered signal to determine the direction of the eye ball and the Figure 6 (b) shows the algorithm to compare it with a user defined threshold. Figure 6 (c) corresponds to the algorithm for acquisition of the electroencephalogram from Neurosky Mindwave headset which determines the user's attention level and compares it with a threshold to activate the motor to move forward. The Figure 6 (d) in the indicator of the proposed algorithm to control motorized system wirelessly with Bluetooth technology. 


\section{International Journal of Innovative Research in Science, Engineering and Technology}

(An ISO 3297: 2007 Certified Organization)

Vol. 5, I ssue 8, August 2016

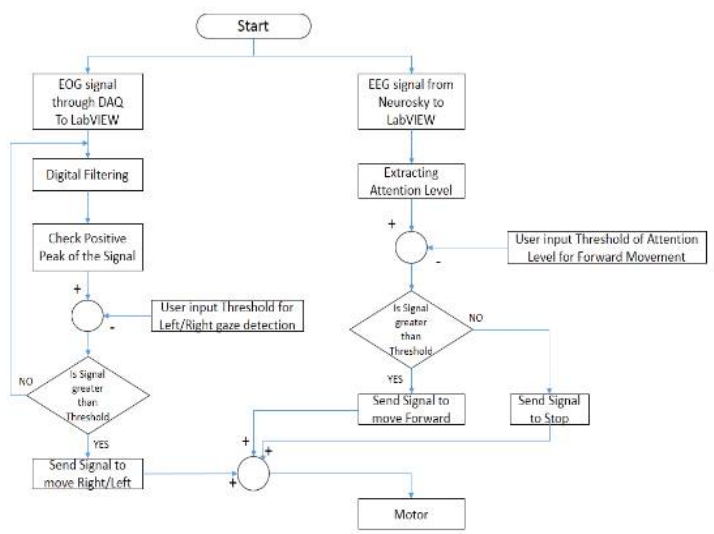

Figure 7: Flow Chart of the Designed System

The above Figure 7 corresponds flow chart of the entire operation. In this flow chart the methodology is described using some decision making steps.

\section{RESULTS}

In this section the snapshots of the front panel, the detection of the gaze and the movement of the car is tried to be shown. The Figure 8 (a) corresponds to the front panel of the vi which was indicating that right gaze has been detected and also as the attention level doesn't crosses the threshold thus the start-stop led is off which means that the motor was stationary and only turned towards right.

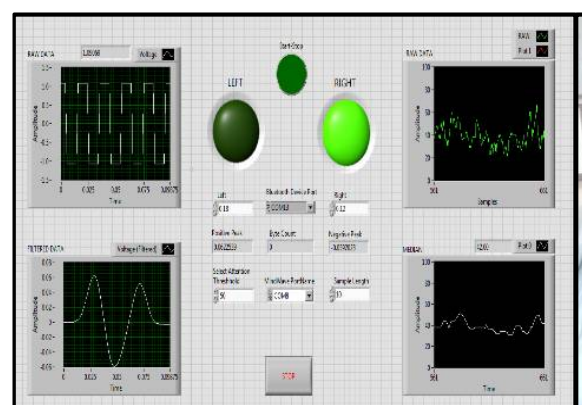

(a)

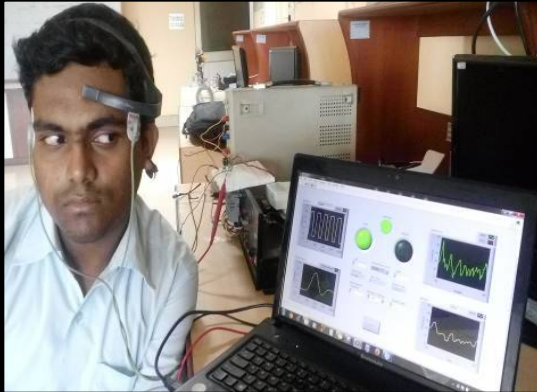

(b)

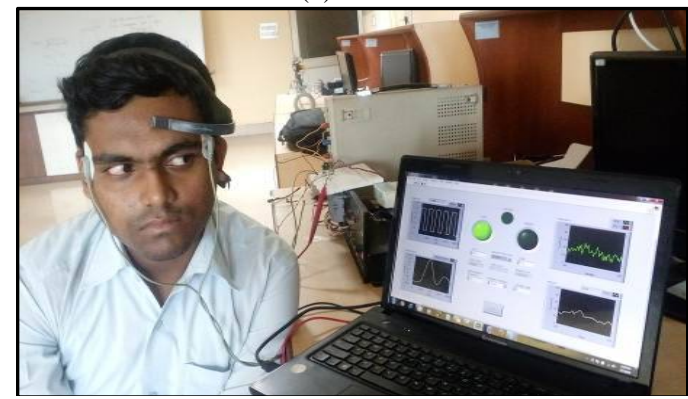

(d)

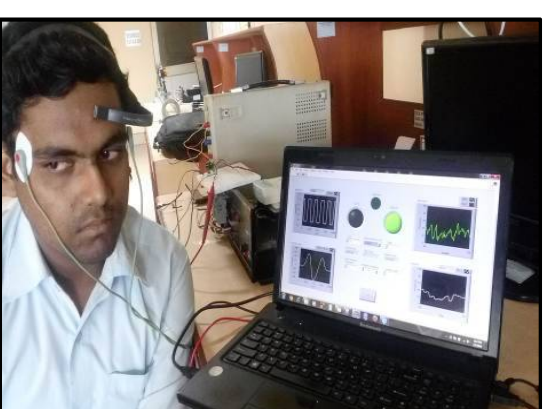

(c)

Figure 8 Front Panel of the System (a) Looking Left while Paying Attention (b)Looking Right Figure (c) Looking Left (d) 


\title{
International Journal of Innovative Research in Science, Engineering and Technology
}

\author{
(An ISO 3297: 2007 Certified Organization)
}

Vol. 5, I ssue 8, August 2016

The Figure 8 (d) indicates the detection of left gaze and Figure 8 (c) indicates the detection of the right gaze. The Figure 8 (b) indicates that there is significant level of attention and also the eyeball is tracked towards left direction which caused the motor to first turn left and then move forward.

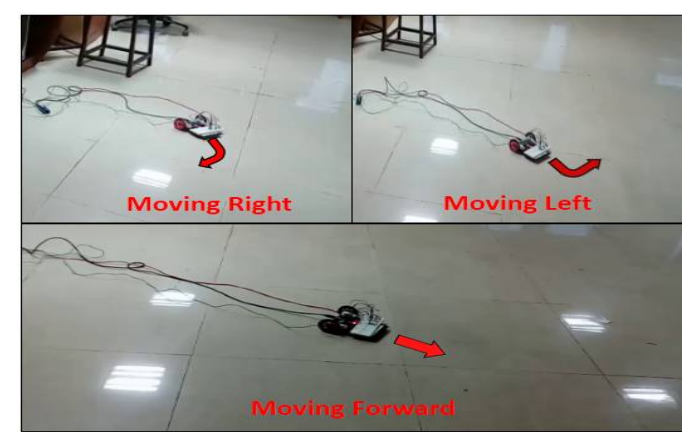

Figure 9: Car Manoeuvring

The Figure 9 corresponds to the different movement of the motorized system in accordance with eyeball movement and attention level.

\section{CONCLUSION}

An eye-gaze interfacing with automated systems would be a new technique than the traditional one. Traditionally, disabled people who cannot move anything except their eyes use eye gaze interaction to move systems using the potentials generated within the body itself. The most significant additionto this work is to control the forward movement and stopping of the system through Mindwave. The effort of designing the system is to direct the motion totally by the eyes and thought process of mind. Due to advancements in the design of eye tracking systems and high processing speed, it can be used for the normal persons also. Eye-tracking interfaces could speed up the interaction, as the eyeball movements are quick. In environments with high hygienic demands such as an operation room for surgery, an eye-gaze interface would be useful because it allows interacting without touching anything [1]. For the improvement of running any electronic system thought control using Mindwave is applied with highest efficiency.

\section{FUTURE SCOPE}

This small prototype of eye tracked and mind operated motorized system can further be implemented in wheelchair for paralysed patients thus making them self-independent and mobile. Also in this era or wearable electronic devices, a headband [11] can further be designed that can have a potential to measure both these parameters thus making it less bulky, less costly and a easy to handle user end device.

\section{ACKNOWLedgMent}

The authors would like to express their gratitude to the TEQIP phase-II, Sub-component 1.2, Narula Institute of Technology, for supporting the research work financially. Additionally authors are very much thankful to EIE Dept. for their cooperation.

\section{REFERENCES}

[1] Dr. Jaswinder Singh, "Human Eye Tracking and Related Issues: A Review", Hari Singh1, International Journal of Scientific and Research Publications, Volume 2, Issue 9, September 2012 ISSN 2250-3153

[2] Andreas Bulling,Student Member, IEEE, Jamie A. Ward, Hans Gellersen, and Gerhard $\operatorname{Tr}$ " oster, Senior Member, "Eye Movement Analysis for Activity Recognition Using Electrooculography", IEEE transactions on pattern analysis and machine intelligence, - preprint 


\title{
International Journal of Innovative Research in Science, Engineering and Technology
}

\author{
(An ISO 3297: 2007 Certified Organization)
}

Vol. 5, Issue 8, August 2016

[3] Chongshi Yue, "EOG Signals in Drowsiness Research", 840220-8139 LiTH-IMT/MASTER-EX--11/007-SE

[4] Jobby K. Chacko, Deepu Oommen, Kevin K. Mathew, Noble Sunny, N. Babu, "Microcontroller Based EOG Guided Wheelchair", World Academy of Science, Engineering and Technology, International Journal of Medical, Health, Biomedical, Bioengineering and Pharmaceutical Engineering Vol:7, No:11, 2013

[5] H Drewes, "Eye Gaze Tracking for Human Computer Interaction", A Dissertation submitted in the partial fulfilment of the Ph. D. Degree, 2010. www.ndltd.org

[6] Kristian Lukander, "Mobile usability - Measuring gaze point on handheld devices" Master's thesis, 2003. www.ndltd.org

[7] H S Dhillon, R Singla, N S Rekhi and R Jha, "EOG and EMG based Virtual Keyboard: A Brain-Computer Interface", 2nd IEEE International Conference on Computer Science and Information Technology, 2009, pp 259-262.

[8] Tobias Wissel1, Ramaswamy Palaniappan, "Considerations on Strategies to Improve EOG Signal Analysis", DOI: 10.4018/jalr.2011070102 - Source: DBLP

[9] Patterson Casmir D’Mello, Sandra D'Souza, "Design and development of a Virtual Instrument for Bio-signal Acquisition and Processing using LabVIEW", International Journal of Advanced Research in Electrical, Electronics and Instrumentation Engineering, Vol. 1, Issue 1, July 2012

[10] Shubhodeep Roy Choudhury, S.Venkataramanan, Harshal B. Nemade, J.S. Sahambi, "Design and Development of a Novel EOG Biopotential Amplifier", IJBEM Vol. 7, No. 1, 2005

[11] Anil P.C, Arvind B, Saritha S, George Varkey, "Development of a Headband for Acquisition and Analysis of Forehead EOG Signal for Driver Fatigue Detection based on Eye-Blink Patterns", International Journal of Computer Applications (0975 - 8887), Volume 96 - No.23, June 2014

[12] M. A. Fkirin, S. Badawy, A. S .El-Sherbeny, (2009) 'Driving a DC Motor by Numerically Manipulated Eye Signal Captured by EOG', The Online Journal on Electronics and Electrical Engineering, Vol. (2) - No. (1) pp. 170.

[13] Rodica Holonec, Marius Gabor, Romul Copindean, Florin Dragan, "An Electooculogram Based Virtual Instrumentation System", Mediamira Science Publisher, Volume 56, Number5, 2015

[14] Mamata Mn, Ramchandran S, Chandrasekharan M, "Smart Sensor Design and analysis of brain machine interface using Labview", IEEE international conference on Communication Software and Networks (ICCSN), pp-464-468, 2011

[15] Gunjeet Kaur, Rashpinder Kaur, "Design and Development of Virtual Instrumentation System for Disabled using LabVIEW", International Journal of Advanced Research in Computer Engineering \& Technology (IJARCET), Volume 2 Issue 11, November 2013

[16] Vijay Raghav Varada, Deepshikha Moolchandani, Asil Rohit, “Measuring and Processing the Brain's EEG Signals with Visual Feedback for Human Machine Interface", International Journal of Scientific \& Engineering Research Volume 4, Issue 1, January-2013, ISSN 22295518

[17] Luzheng Bi, Xin-An Fan, Yili Liu "EEG-Based Brain-controlled Mobile Robots: A survey", Human-Machine Systems, IEEE Transactions on (Volume: 43, Issue: 2), pp. 161-176, Mar 2013.

[18] "Biomedical Instrumentation and Measurements", Leslie Cromwell, Fred J. Weibell, Erich A. Pfeiffer, Second Edition, PHI Learning Private Limited, ISBN:978-81-203-0653-0

[19] R. Robbins, M. Stonehill, "Investigating Neurosky Mindwave EEG Headset", Published Project Report PPR726, Transport Research Foundation, 2014

[20] Neurosky, "Mind-set instruction manual", Available: http://developer.neurosky.com /docs/doku.php?id = mindset_instruction_manual

[21] NeuroSky, I. A. (2010). ThinkGear Socket Protocol (Tech. Rep.). Available from http://www.neurosky.com

[22] Labview User Manual, April 2003 Edition, National Instruments

[23] Micheal, Arduino Cookbook, 3rd ed., vol. 2 